\title{
Implications of mHealth Service Deployments: A Comparison between Dissimilar European Countries
}

\author{
Frederic Vannieuwenborg ${ }^{1}$, Zviad Kirtava ${ }^{2}$, Lambros Lambrinos ${ }^{3}$, \\ Jan Van Ooteghem ${ }^{1}$, and Sofie Verbrugge ${ }^{1}$ \\ ${ }^{1}$ Ghent University, IBBT, Dept. of Information Technology (INTEC) \\ ${ }^{2}$ Partners for Health NGO, National Information Learning Centre \\ ${ }^{3}$ Cyprus University of Technology, Dept. of Communication and Internet Studies \\ \{frederic.vannieuwenborg, jan.vanooteghem, \\ sofie.verbrugge\}@intec.ugent.be, zkirtava@nilc.org.ge, \\ lambros.lambrinos@cut.ac.cy
}

\begin{abstract}
Despite several successful pilot studies, a general implementation and adoption of mobile health services is not often the case. The deployment of eHealth and in particular mHealth services is discussed for three non similar European countries: Cyprus, Georgia, and Belgium. Each country uses the same kind of mobile health services with a different emphasis. By comparing results of a reference case, tele-monitoring heart patients, major implications are identified for a general rollout of services and respective recommendations.
\end{abstract}

Keywords: mHealth, Tele-monitoring, Business model implications.

\section{Introduction}

Driven by an aging population, the European demand for care and cure will strongly increase. Also the growth of medical requirements in remote locations in developing states, lower availability of professional care providers, and all this within a stringent health care budget will force us to change the actual way of providing good quality care. Next to revaluation of the care sector and optimization of the work efficiency, integration of ICT in the care sector already has proven its positive impact.

eHealth, and in particular, one of its service - telemedicine; diagnosis and treatments at a distance - currently finds application in the majority of medical domains: radiology, cardiology, dermatology, psychiatry, dentistry and paediatrics. Telemedicine, offering quality services with significant cost savings, has given a new dimension to the healthcare sector. Various telemedicine programs have proven to be cost effective [3] [23] [24], and with the curtailment of health care expenditures, programs as such are being implemented in several nations [7].

\section{2 eHealth: Actual Implementation}

Two sorts of services should be distinguished. The most obvious mHealth services are those that are regulated. This type of services is developed within a set of regulations 
(e.g., privacy or data presentation) to support the professional care providers with professional data. In contrast, non regulated health services are rising under the form of numerous web- and smart phone applications. They can also provide useful information for the caregivers, but are much less restricted by legal facets.

\subsection{Regulated Services}

eHealth encompasses a range of services or systems that are at the edge of medicine/healthcare and information technology, including (but not limited to):

- Health Management Information Systems (HMIS), Electronic Health/Medical Records (EHR/EMR) and Electronic Health Data Registries,

- Telemedicine: diagnosis and treatments at a distance,

- Health professionals' distance learning and re-training, and

- Health knowledge management through medical informatics resources: e.g., Evidence Based Medicine (EBM) or best practice guidelines on Consumer health/personal informatics, health literacy: use of health/medical e-resources by healthy individuals or patients.

eHealth is seen as recovery and growth tool for healthcare sector by leading economies in the world. The US government has presented a new Strategy for American Innovation on Feb 4, 2011, where Health IT products are named as one of the most important driving forces for improving cost-efficiency and driving US economy towards sustainable growth. According to IDC Research (2008) Western EU market for eHealth will grow from $\$ 9$ billion (in 2006) to $\$ 12$ billion (in 2011). A similar trend of doubling - from $\$ 16$ billion to $\$ 35$ billion - is foreseen for the US Health IT market. Other continents like Asia and Europe also stimulate heavily the implementation of eHealth applications in order to curtail governmental expenditure on healthcare services [21].

In developing economies or countries with many widespread inhabitants, eHealth applications (e.g. telemedicine, tele-learning or tele-radiology) play an important role to bridge the gap of medicine between rural and urban areas. Typically medical facilities in rural domains do not always offer the same quality level as urban healthcare centers [7].

Mobile Telemedicine (Mobile Health, M-Health) represents a rapidly growing branch of telemedicine services and more specific, providing continuous medical care (at-home and outdoors) preferably based on wireless sensors and actuators communicating with different health actors (hospital specialist, General Practitioner, Medical Insurance Company) via GPRS, 3G or LTE connections. Thanks to the growing use of mobile phones, increase in network coverage, bandwidth rich applications, technical improvements, financial affordability of broadband applications and the lower tariffs for data transmission, mobile telemedicine is evolving as an advantageous and cost-effective tool. The overall objective of mobile telemedicine is the development and trial of new services and applications in the area of mobile health, promoting the use and deployment of GPRS, 3G or LTE mobile services and technologies. The target is to provide the means contributing to the 
reduction of costs in hospitals and health care, by allowing patients to have complete and personalized monitoring of their health in-home, while pursuing a normal life (instead of being confined in hospital for long periods of monitoring).

Most efficient mHealth applications regard remote monitoring of mainly outpatients with different chronic conditions which cannot be treated at wards continuously but would benefit by having extended remote monitoring:

- Diabetes patients monitoring

- Patients with risk of blood clotting (myocardial infarction, transient ischemic attack /stroke, deep veins thrombosis, pulmonary artery thromboembolism)

- Bronchial Asthma patients

- Arterial hypertension

- Cardiac Arrhythmias

- Epilepsy

- Chronic Heart Failure

\subsection{Non-regulated Services}

Next to all regulated forms of eHealth and mHealth applications (approved and adopted by (un)professional caregivers and regulators) also unregulated applications, under the form of Internet- and smart phone applications, are expected to gain importance in the care sector. With the growing use of smart phones and all kind of sensor devices, the number of personal informatics services, which are applications allowing people to track their own habits, movements and thoughts, grows exponentially [14] [17]. According to the Global Mobile Health Market Report 20102015 [15], in 2015 more than 500 million people will have a mHealth application on their smart phone. The resulting data of these activities such as your personal sleeping pattern, your heartbeat during an exercise, your daily weight, will be an unregulated, but useful completion for a personal health record [15]. Some cardiologists are already tracking patients conditions based on data provided by a Polar ${ }^{\circledR}$ application.

\section{Reference Case: Heart Failure Monitoring}

Because there already exist numerous projects [4] [20] [2] performed all over the world, which monitored patients with heart failures, we choose to use this mobile application as reference case. This pool of available data allows us to form a good image of the operational processes, the financial and juristic characteristics of mobile monitoring of heart failure patients.

With the actual available technology, mobile monitoring of heart failure patients is relatively easy to do. Generally the infrastructure consists of a central server and/or a call center (Figure 1), a mobile phone and medical devices (an Electrocardiogram (ECG) device, a weighing scale and a monitor for the blood pressure) suited for monitoring arrhythmia/heart failures. A patient can monitor his/her vital body parameters regularly from anywhere (e.g., home or office) and the data is transferred to the server via the mobile phone or gateway. The server analyses the data for any 
'red alerts' or 'technical alerts'; also readings are stored in the patients' personal electronic records in the server. In case of any emergency, an alert is sent to the GP, nurse and/or cardiologist, who can access the health records via mobile phone or computer connected to Internet, and correctly diagnose, prescribe additional medicine, take action.

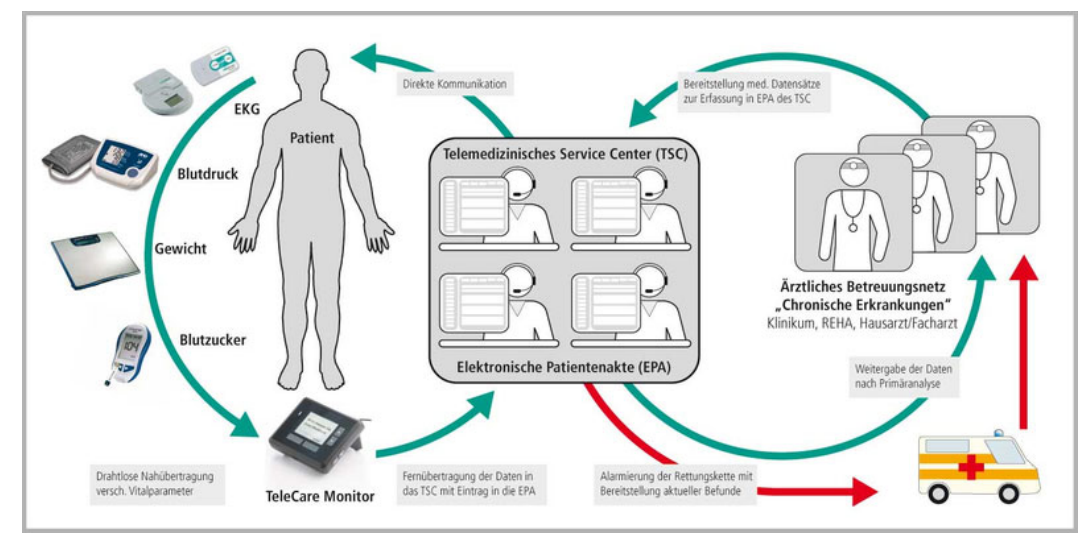

Fig. 1. Tele-monitoring patients with heart failures, source [6]

Tele-monitoring creates a win-win situation for the patient, doctor, hospital and insurance provider [20]. A patient is reassured that his/her health is in good hands, by being silently monitored all the time. Together with better disease education and acceptation, what grows noticeable, this leads to immediate improvement of the quality of life for the patient. Doctors can pursue other activities or manage different patients. At the same time they still can be reached easily anywhere and anytime. Also, historical data can reveal insights in the development of the disease. This could be a valuable input for future research and development in the pharmaceutical and medical field. Hospitals can discharge patients earlier, and insurance companies do save funds with reduced number of hospitalizations and shorter in-hospital (ER or specialized department) stays [4] [6] [1].

\section{Cases}

In what follows we describe a general overview of the 'as is' state in Georgia, Belgium and in Cyprus. By doing so, we hope to identify some of the implications for a generalized rollout of this telemedicine application.

\subsection{Actual Status: Georgia}

Georgia is a developing low and middle income country that recently reformed a part of its healthcare delivery system. It changed from a universal low-cost healthcare model that was inherited from the Soviet Union, to a largely fee-for-service system. 
Cardiovascular diseases, especially Arterial Hypertension (AH) and Ischemic Heart Disease (IHD) are the main cause of both morbidity (mainly AH) and especially mortality (mainly IHD) in Georgia.

According to statistics provided by World Health Organization [25], in 2002 Georgia was among the five leading countries (Bahamas, Georgia, Lesotho, Liberia and Maldives) with the highest incidence of AH. Based on the WHO Surveillance of Chronic Risk Factor Report [28] for 2005, adults from Georgia had the second highest average blood pressure in the world. According to most recent statistics provided by Georgian National Center for Disease Control and Public Health, the incidence of AH in Georgia reached 55\% by the end of 2009 exceeding the alarming incidence of high blood pressure (43\%) as found for African Americans [27].

One of the major complications of both AH and chronic IHD is heart failure. In order to anticipate these risks, they imported a heart failure disease management program (HFMDP) to better follow up and start monitoring their chronic heart failure patients. Results of this implementation are promising less emergency department visits and hospitalizations. On the other hand, more Beta-blockers are prescribed [8].

Finally, one of the leading causes of sudden death and patient disability among cardiology patients are cardiac arrhythmias. Regretfully, until now, Georgian health statistics do not register separately numbers of arrhythmias. However, by approximation of European data we can estimate that there are around 70,000 patients with arrhythmia annually in Georgia. Those patients often need ambulatory monitoring and/or hospitalization. However, home monitoring is not included in insurance schemes, whilst hospital in-patient costs are very high (around 40-110 euros daily, in a country with an average salary still below 100 euros per month).

Georgia had its first eHealth application in cardiology in 2000-2001, when coronarography files of patients with chronic IHD and acute coronary syndromes, like Miocardial Infarction were transferred from Chapidze Emergency Cardiology Center (ECC) for second opinion to Bad Krozingen Heart Center (Germany). The selected patients, who had to undergo not the easier stenting procedure (already available in Tbilisi) but the more serious coronary bypass surgery, were then sent to Germany until 2002. After that, the operation also became routine at CECC [9].

Another project, the first mHealth service in Georgia and funded by a local branch of US CRDF (Civilian Research and Development Foundation) and local NSF (National Science Foundation) funds, has tested the feasibility of tele-monitoring cardiac arrhythmia for patients outside the hospital [10]. The Vitaphone (Germany) ECG loop recorder and special software pre-recorded GSM phones have been used [6]. The local mobile operator provided his services for free for the arrhythmia monitoring cases. The ECG Loop Recorder automatically recorded arrhythmia events, which were transmitted via Bluetooth to the mobile phone and then by $3 \mathrm{G}$ or GPRS (partner mobile operator - MagtiCom Ltd.) to the Vita server in Germany and available to Georgian physician via e-mail/Internet. Cases of sinus brady- and tachyarrhythmia, sinus node weakness syndrome, atrial fibrillation, supraventricular tachycardia, supraventricular premature complexes (SPCs) and ventricular premature complexes (VPCs) have been correctly recognized by automatic recognition software and recorded. Patient observations by mobile telemedicine turned out $2.5-5$ times less 
expensive than keeping them at cardiac wards. $85 \%$ of the patients have assessed the technology as very comfortable.

In a second stage, the above-mentioned project aims to be extended to Arterial Hypertension (2012) and later - to Chronic Heart Failure patients. In addition, starting from 2012, eHealth applications for cardiac patients will be provided to the Adjara Healthcare system in order to stay connected with some mountain villages that are out of reach in winter due to heavy snow. For these villages, tele-consultations are extremely important.

Relative challenges for business applications are related to high hardware cost and yet a needed paradigm shift in insurance schemes. In general, insurance companies have the policy to cover high inpatient costs, but are hesitating so far to support outpatient monitoring costs. The issue is discussed with the Parliamentary commission on health and social care and some changes are expected. Another issue is the fee for physicians who monitor the patient's ECG 24 hour. So far only four centers have enrolled in the program and due to relatively low number of patients, costs for services per participant, especially without insurance support, are high.

\subsection{Actual Status: Belgium}

Although the population of Belgium is rapidly aging and pressure on the care sector is still rising, this developed Western European country has not succeeded yet to implement any kind of generally accepted and applied model of a heart failure monitoring application. The main causes of death are also related, as practically all Western Countries, to cardiovascular problems. The big issue is that the number of cardiovascular patients grows exponentially starting from the age of 60 [5].

Now policy makers realize that tele-monitoring applications can be a key answer to the challenge of Belgium's aging, and more care demanding population. We noted that several projects, involving tele-monitoring patients, were done or are running at this moment [18]. Five projects are handling about monitoring heart failure patients; two of them are already finished. Both studies proved better quality of life for the patient and reduced cost for the health insurances [4].

After interviewing cardiologists who led the projects, we had to conclude that the demand for monitoring heart failure patients is huge: 15000 new patients a year in Belgium. The method proved to be efficient. There are several hardware manufacturers and distributors, and the care sector itself that is thrilled to deploy such an application. Then why didn't a general rollout happen yet? It seems that the answer can be found in the actual business model. Today, tele-monitoring applications aren't recognized by the insurance companies as an official manner to monitor a patient's health condition. So except when one joins a research project, all the hardware has to be bought by the patient or his/her family. Next to that, the professional care givers need time to analyze the results when an alarm is triggered or generated without being paid for it. In fact they lose income because in that time, other patients could have been examined. Another fundamental question that still needs to be answered handles about who needs to analyze and monitor the results. A GP has a more trust based relationship with his/her patients, but has normally only a few of chronic heart failure 
patients. So one can think that a specialized team of heart failure nurses, which can also build a sort of trust relationship with the patients, is more efficient then a GP, but then a GP loses contact with his patients, so an intermediate solution should be created.

So in order to implement a generally accepted heart failure tele-monitoring application one important final step should be made. More investigation is needed for the individual business models for the actors in order to achieve a social optimum. There has to be a way that the system is affordable and that all actors involved earn according to their involvement. The federal institution for healthcare insurance (RIZIV) is now working on the rollout of a so called care path. A care path is an individual approach for offering the right kind of healthcare to a patient. In this system there is an incentive foreseen per patient for all professional actors involved such as the GP, the cardiologist, hardware seller.

\subsection{Actual Status: Cyprus}

In Cyprus, there are various activities that utilize modern telecommunication technologies in an attempt to improve health-related services. These telemedicine applications range from home monitoring of patients to the enhancement of emergency response services.

In the home monitoring front, the focus is on children with cardiac arrhythmias which constitute one of the most difficult problems in cardiology both in terms of diagnosis and management. As said, the continuous monitoring of ECG vital signs and environmental conditions can significantly improve the chances of identifying possible arrhythmias. The outcome of this work is the prototype of a system [12] that is specifically designed for children and is able to carry out the acquisition and transmission of ECG signals. The fact that data transmission takes place in real-time allows for the deployment of an alarm-type scheme that assists in the identification of possible arrhythmias by notifying the on-call doctor and the child's relatives when abnormal events take place.

Turning the focus to emergency health care support, one activity aims in enhancing support to medical staff handling an emergency situation. The system developed [11] is a combined real-time and store and forward facility that consists of a base unit and telemedicine (mobile) units. While at the emergency site (e.g., ambulance, rural health centre, or ship) the mobile telemedicine unit communicates with a base unit at the hospital-expert's site. The system allows for the real time transmission of vital biosignals (3-12 lead ECG, SPO2, NIBP, IBP, and temperature) and still images of the patient. Its use was demonstrated and tested in Greece, Cyprus, Italy and Sweden [16]. The system is deployed in the district of Paphos in Cyprus: a rural health center, a small hospital and an ambulance are all connected to the Paphos General Hospital.

To provide further assistance in emergency situation handling, other efforts concentrate on enhancing the response times of the emergency service teams in cases of new incidents since in a number of cases any unnecessary delay may prove to be critical. In an attempt to start incorporating features that relate to Next Generation 112/911 services, smart call management mechanisms have been suggested [13] that 
allow the PSAP (Public Safety Answering Point) to apply call priority policies (e.g. for calls coming in from new numbers). Through such smart call handling algorithms and exploiting the features of today's smart phones for exact location identification one may reduce the response time to an incident. Moreover, analyzing past calling history in combination with location information can provide an estimate on the extent of an incident thus allowing for better resource planning and allocation.

The solutions described so far operate on the assumption that a modern telecommunications infrastructure is in place and constantly operational. Unfortunately, this is not the case worldwide and especially in some rural areas and large parts of developing countries. To allow for the transfer of telemedicine data under such conditions, a system [19] has been devised that operates according to the store-and-forward principle utilized in Delay Tolerant Network (DTN) deployments. The particular implementation concentrates on the transfer of low volume data that can be quickly read or easily keyed-in on a telephone keypad by a patient or caregiver and is not time critical e.g. scheduled blood pressure readings.

The potential outcomes of the work described in this section are highly promising and this has been demonstrated through small scale deployments. However, to date, a country-wide deployment of a telemedicine-related platform is still not present.

\section{Comparison between Countries}

Georgia, Belgium and Cyprus and most Western Countries would profit immensely by rolling out a tele-monitoring application for heart failure or cardiac arrhythmia patients. But emphasizes are different.

- In Belgium, already a backbone of care infrastructure for heart failure patients is foreseen, such as specialized heart failure hospital departments. The main reason of developing such a service would be to lower the cost for healthcare system and increase the quality of life.

- In Georgia, the health sector is still under revision. The primary goal is to reach people in rural areas and to provide stimuli to develop primary health care and outpatient tele-monitoring. Lowering costs is important, but to do that efficiently, medical management programs (such as HFMDP, Arterial Hypertension Control program) should be generalized and optimized first.

- Cyprus lies in between. A dedicated heart center is not a reality yet, but they're integrating one. On top of that, according to [26], they tend to possess a lot of expertise and awareness of the possibilities of eHealth and mHealth.

It may be concluded that, for these three countries, 'the last mile' is the major drawback to implement mHealth applications to monitor the heart condition. Although several projects proved to have successful results, Belgian, Cypriot and Georgian health insurers tend to have a very cautious view. They are willing to pay huge amounts of money to inbound patients in hospitals, but a much more economical solution such as tele-monitoring is not paid back. Fortunately, negotiations with small private insurers are taking place. Hopefully this is a trigger for other, more incumbent, 
health insurance companies. Once a good acceptance is created, other mHealth applications can be implemented as well.

\section{Conclusions and Lessons Learned}

The following conclusions can be drawn:

- The reference case showed that all countries (less/well developed) can gain by implementing a tele-monitoring service. Not only for lowering costs, but also for improving population's access to healthcare services.

- A sustainable business model that takes into account all the individual interest of the actors involved, professional and unprofessional (Cardiologist, general practitioner, heart failure nurses, health insurance companies, policy makers,...) is the key to a generally accepted tele-monitoring program [22].

- There exists a huge market potential for tele-monitoring services. In order to support this growth, actors like mobile operators, health insurance companies, hardware and application developers, etc. should further innovate and adapt their actual products. Next to these regulated services, non- regulated applications in the form of smart phone and internet applications will also become a part of our daily life.

In general can be said that the introduction of smart ICT and Future Internet services, such as video telephony, telemedicine, tele-monitoring, and personal alarm systems can contribute to a more sustainable and qualitative life for the whole population with emphasize on the elderly people living at home. This involves a lot of actors in a complex eco-system, all with their own expectations and outlook for potential benefit, either in business or social benefit.

That is the reason why it is so important to succeed in implementing the telemonitoring service for heart failure patients. All necessary structures are already available, it's 'just' a matter of formulating a sustainable business model for all actors and the resulting Snowball effect will speed up the implementation of all following mHealth applications.

Open Access. This article is distributed under the terms of the Creative Commons Attribution Noncommercial License which permits any noncommercial use, distribution, and reproduction in any medium, provided the original author(s) and source are credited.

\section{References}

1. Dadoo, V.: White Paper on Mobile Health (2006), http://www.interactwar e.com/wp-content/uploads/2011/08/White-paperMobile-Healthcare-2006.pdf

2. Dang, S., Dimmick, S., Kelkar, G.: Evaluating the Evidence Base for the Use of Home Telehealth Remote Monitoring in Elderly with Heart Failure. Telemedicine and e-Health 15, 783-796 (2009) 
3. Dansky, K.H., Palmer, L., Shea, D., Bowles, K.H.: Cost Analysis of Telehomecare. Telemedicine Journal and e-Health 7, 225-232 (2001)

4. Dendale, P.: Intensive telemonitoring program of patients with chronic heart failures (2010), http://www.jessazh.be/deelwebsites/Hartcentrum_Hasselt/ Wetenschappelijke_info/Cardiologisch_congres_2010/

Cardio_2010_Dendale_Telemonitoring_in_hartfalen

5. K. M. O. FOD Economie, Middenstand en Energie, Causes of Death, statistics for 2006, http://statbel. fgov.be/nl/binaries/ NL $\% 20-\% 20 \mathrm{Tableau} \% 201.3 \_T \_t c m 325-138394 . p d f$

6. Fransen, E.: The Vitaphone Tele-ECG System: Event Recording in Patients With Arrhytmias (2009), http://www.slideshare.net/guestf9a3fbf/vita phone-tele-ecg-system-presentation

7. I. Global Industry Analysts, Global Telemedicine Market to Exceed \$18 Billion by 2015 (2009)

8. Hebert, K., Quevedo, H.C., Gogichiashvili, I., Nozadze, N., Sagirashvili, E., Trahan, P., Kipshidze, N., Arcement, L.: Feasibility of a Heart Failure Disease Management Program in Eastern Europe: Tbilisi, Georgia. In: Circulation: Heart Failure, September 7 (2011)

9. Kirtava, Z., Aladashvili, A., Chapidze, G.: Still Image Teleconsulting for Interventional Cardiology in Georgia. Presented at the Abstracts of the 6th Int. Conference on Telemedicine, vol. 9, pp. 342-343 (2001)

10. Kirtava, Z.: e-Health and $\mathrm{m}-\mathrm{Health}$ : Trends and visions for resource-limited environment in developing economies - case of Georgia. Presented at the ESF and COST High-Level Conference "Future Internet and Society - Complex Systems Perspective", Acquafredda di Maratea, Italy (2010)

11. Kyriacou, E., Pavlopoulos, S., Berler, A., Neophytou, M., Bourka, A., Georgoulas, A., Anagnostaki, A., Karayiannis, D., Schizas, C., Pattichis, C., Andreou, A., Koutsouris, D.: Multi-purpose HealthCare Telemedicine Systems with mobile communication link support. BioMedical Engineering OnLine 2 (2003)

12. Kyriacou, E., Pattichis, C., Hoplaros, D., Kounoudes, A., Milis, M., Jossif, A.: A System for Monitoring Children with Suspected Cardiac Arrhythmias - Technical Optimizations and Evaluation. In: Bamidis, P.D., Pallikarakis, N. (eds.) XII Mediterranean Conference on Medical and Biological Engineering and Computing, vol.29, pp. 924-927. Springer, Heidelberg (2010)

13. Lambrinos, L., Djouvas, C.: Using location information for sophisticated emergency call management. In: Lin, J.C., Nikita, K.S. (eds.) MobiHealth 2010. LNICST, vol. 55, pp. 227-232. Springer, Heidelberg (2011)

14. Lee, I.: in personalinformatics.org, ed: HCII, Carnegie Mellon University (2011)

15. Mikalajunaite, E.: $500 \mathrm{~m}$ people will be using healthcare mobile applications in 2015 , October 19 (November 2010), http://www.research2guidance.com/500mpeople-will-be-using-healthcaremobile-applications-in-2015/

16. Mougiakakou, S., Kyriacou, E., Perakis, K., Papadopoulos, H., Androulidakis, A., Konnis, G., Tranfaglia, R., Pecchia, L., Bracale, U., Pattichis, C., Koutsouris, D.: A feasibility study for the provision of electronic healthcare tools and services in areas of Greece, Cyprus and Italy. BioMedical Engineering OnLine 10, 49 (2011)

17. Quantified Self (2011), http: / /quantifiedself.com/guide/tag/gadget/reviews / 4

18. RIZIV, Telemonitoring voor chronische patiënten : financiering van 6 projecten, October 18 (2011),

http://www.riziv.be/care/nl/infos/telemonitoring/index.htm\#2 
19. Scholl, J., Lambrinos, L., Lindgren, A.: Rural Telemedicine Networks using Storeandforward VoIP and DTN. Presented at the Medical Informatics Europe (MIE 2009), Sarajevo, Serbia (2009)

20. Seto, E.: Cost Comparison Between Telemonitoring and Usual Care of Heart Failure: A Systematic Review. Telemedicine and e-Health 14, 679-686 (2008)

21. Valeri, L., Giesen, D., Jansen, P., Klokgieters, K.: Business Models for eHealth. Presented at the ICT for Health Unit, DG Information Society and Media (2010)

22. Van Ooteghem, J., Ackaert, A., Verbrugge, S., Colle, D., Pickavet, M., Demeester, P.: Economic viability of eCare solutions. In: eHealth 2010: Proceedings of the Third International ICST Conference on Electronic Healthcare for the 21st Century (2010)

23. Whited, J.D.: Economic Analysis of Telemedicine and the Teledermatology Paradigm. Telemedicine and e-Health 16, 223-228 (2010)

24. Winblad, I., Hämäläinen, P., Reponen, J.: What Is Found Positive in Healthcare Information and Communication Technology Implementation?- The Results of a Nationwide Survey in Finland. Telemedicine and e-Health 17, 118-123 (2011)

25. World Health Organization, World Health Report 2002: Reducing risks, promoting healthy life. WHO Press, Geneva, Switzerland (2002)

26. World Health Organization, Surveillance of chronic disease: risk factors: countrylevel data and comparable estimates (SuRF reports; 2). WHO Press, Geneva, Switzerland (2005)

27. World Health Organization: Global Observatory for eHealth series 1 (2010), http://www.who.int/goe/publications/ehealth_series_vol1/ en/index.html

28. World Health Organization Regional Office for Europe, Assessment of NCD prevention and control in Primary Health Care, Georgia (2009) 